\title{
Research of Multiple Sensors Adaptive Fault-Tolerant Control Based on T-S Fuzzy Model for EMB System
}

\author{
Y. N. Xu and W. W. Deng
}

\begin{abstract}
In the electro mechanical brake (EMB) system, an improvement of the fault-tolerant control, like invalid protections of sensors and electric systems are the key problems to the development of electric vehicles. The adaptive fault-tolerant control in this paper is focus on the descriptor nonlinear system which contains double time-delays and parameter uncertainties. In fault detection and estimation, construct the controlled system model which contains multi-sensor, double time-delays and parameter uncertainties based on T-S fuzzy model, then design the observer to realize fault detection and estimation in real-time. In building the fault-tolerant control model, the first step is to choose an appropriate sliding surface, and combine the algorithm of sliding control with adaptive generic model control. Then apply the state observer to the designed sliding adaptive generic model, and build the decision model with dynamic fault reconfiguration. And the goal of this paper about the adaptive robust fault-tolerant control for the complex nonlinear controller system is achieved. Numerical SIMULINK simulation examples are given to illustrate the application and the effectiveness of the proposed design method.
\end{abstract}

Index Terms-Electro mechanical brake, sensors, T-S fuzzy model, adaptive fault-tolerant control, sliding model control.

\section{INTRODUCTION}

Sensors are main tools to percept, detect, and obtain messages. There are several sensors loaded on the electronic brake pedal simulators and actuators in EMB on the purpose of detecting current, speed, pressure, acceleration, and angular velocity etc. Sometimes, these sensors could occur relaxing, interval, and abrupt faults. As the braking system is essential for automotive safety, once the EMB system has fault, it will cause a major threat to vehicle safety [1], [2]. Faults could be caused by external environments like the electromagnetic field induced by a loaded high-power motor, the vehicle vibration, temperature changes, as well as a lifetime of the electronic components. Once any devices in EMB system has fault occurs, the braking system will misunderstand the driver's braking intention, and cause serious problem [3], [4]. Thus, make the EMB system which has satisfy output even as fault occurs is necessary. The adaptive fault-tolerant technology is an important method to improve the safety and reliability of the system which is widely used in numerical control field. Actually, general

Manuscript received April 3, 2014; revised May 28, 2014. This work was supported by the Jilin University of State Key Laboratory of Automotive Simulation and Control open fund (20121120) and National Natural Science Foundation of China (61361003).

The authors are with the Jilin University, Changchun, China. And they are also with of Yanbian University, Yanji, China (e-mail: ynxu@ybu.edu.cn, kdeng@jlu.edu.cn). controlled systems show properties like nonlinear, time-varying, disturbances, and uncertainties, the accurate mathematical model is difficult to be constructed. Takagi and Sugeno have mentioned the T-S fuzzy model method which is used to describe complicated nonlinear system [5]. Then Taniguchi T. proposed T-S fuzzy descriptor system model in 1999 which can transfer the nonlinear system to multiple topical linear system, simplified the control process and improve the control accuracies [6]. In order to solve the time-delay problem of sensor fault, Gao etc. published the fault-tolerant control method where the observer is designed through constructing a linear descriptor system [7]. Most of previous researches are focused on system model, fault model, time-delays, and stability analysis based on the given controlled system (eg. the sensor or the actuator). On the other hand, there are few researches about multi-target global nonlinear descriptor system considering multi controlled objects at the same time.

Usually, designs in the nonlinear adaptive fault-tolerant systems are based on nonlinear process model and nonlinear controllers. The Kalman filter is a traditional adaptive model that shows unsatisfied control effects because of its poor robustness against model errors [8]. The sliding model control is an effective control method for nonlinear systems with advantages like fast responses, good transient performances, the excellent robust, and the anti-perturbation. Therefore, it can improve the robustness and reliability of the controlled system [9], [10].

In this paper, the adaptive fault-tolerant control method based on T-S fuzzy model for EMB system with multiple sensor faults is proposed. Then, the adaptive generic model control (AGMC) is improved by combining sliding model control (SMC). Finally, the fault-tolerant dynamic decision architecture is constructed based on SMC-AGMC algorithm.

\section{T-S FUZZY DESCRIPTOR SYSTEM MODEL}

Fault detection is the basis of the fault-tolerant control. In fault detection and estimation, the main work is to establish the fault estimation model. In this section, the first step is to construct the EMB system model which contains double time-delays and parameter uncertainties based on T-S fuzzy model. Then design the observer which can estimate system states and faults in real-time.

T-S fuzzy descriptor system model is usually described by a series of fuzzy condition sentences, and each fuzzy model rules can represent one subsystem. Considering describe the descriptor system with input delay, state delay, and parameter uncertainties by T-S fuzzy model. Setting $\mathrm{R}_{\mathrm{i}}$ as the fuzzy model rule of the T-S fuzzy descriptor system, then the i-th 
fuzzy model rule can be described as follows:

$$
\begin{aligned}
& R_{i}: \text { If } \xi_{1}(t) \text { is } M_{1 i} \text { and } \xi_{p}(t) \text { is } M_{p i}, \text { then } \\
& \begin{aligned}
\dot{x}(t)= & {\left[A_{i}+\Delta A_{i}(t)\right] x(t)+\left[A_{i d}+\Delta A_{i d}(t)\right] x(t-d) } \\
& +\left[B_{i}+\Delta B_{i}(t)\right] u(t)+\left[B_{i d}+\Delta B_{i d}(t)\right] u(t-\tau) \\
y(t)= & C_{i} x(t), \quad i=1,2, \cdots, r
\end{aligned}
\end{aligned}
$$

where, $M_{p i}$ is the fuzzy set, $r$ is the number of fuzzy rules; $\xi_{1}(t) \cdots \xi_{p}(t)$ are the premise variables assumed measurable; $x(t) \in R^{n}$ is the state vector, $u(t) \in R^{n}$ is the input vector; $y(t) \in R^{n}$ is the measurable output vector; $\mathrm{d}$ is the state time-delay and $\tau$ is the input time-delay. $A_{i}, A_{i d}, B_{i}, B_{i d}$, and $C_{i}$ are the known constant matrices with appropriate dimensions. $\Delta A_{i}(t), \Delta A_{i d}(t), \Delta B_{i}(t)$, and $\Delta \mathrm{B}_{\text {id }}(\mathrm{t})$ are the parameter uncertainties and considered norm-bounded, in the form of $\left[\begin{array}{llll}\Delta A_{i} & \Delta A_{i d} & \Delta B_{i} & \Delta B_{i d}\end{array}\right]=H_{i} F_{i}(t)\left[\begin{array}{llll}E_{1 i} & E_{2 i} & E_{3 i} & E_{4 i}\end{array}\right]$. where, $H_{i}, E_{1 i}, E_{2 i}, E_{3 i}$ and $E_{3 i}$ are the known real constant matrices with appropriate dimension. $F_{i}(t)$ is an unknown matrix function satisfying $F_{i}(t)^{T} F_{i}(t) \leq I$, here $I$ is the identity matrix of appropriate dimension.

Since the braking of electric vehicles is induced by sensor faults in the EMB system, multiple sensor faults could be considered as states which are contained in the EMB system. Then, the augmented system without containing fault states is proposed. Through processes of center-average defuzzifier, product interference, and singleton fuzzifier, the whole augmented T-S fuzzy system could be inferred as follows:

$$
\begin{gathered}
\dot{x}(t)=\sum_{i=1}^{r} q_{i}(\xi(t)) \cdot\left\{\begin{array}{l}
{\left[A_{i}+\Delta A_{i}(t)\right] x(t)+\left[A_{i d}+\Delta A_{i d}(t)\right] x(t-d)} \\
+\left[B_{i}+\Delta B_{i}(t)\right] u(t)+\left[B_{i d}+\Delta B_{i d}(t)\right] u(t-\tau)
\end{array}\right\} \\
q_{i}(\xi(t))=\frac{w_{i}(\xi(t))}{\sum_{i=1}^{r} w_{i}(\xi(t))} \geq 0, \\
w_{i}(\xi(t))=\prod_{j=1}^{p} M_{l}^{i}(\xi(t)) \geq 0, \sum_{i=1}^{r} q_{i}(\xi(t))=1
\end{gathered}
$$

where, the augmented matrixes are shown as follows:

$$
\begin{gathered}
\bar{E}_{i}=\left[\begin{array}{cc}
E_{i} & 0 \\
0 & 0
\end{array}\right], \bar{A}_{i}=\left[\begin{array}{cc}
A_{i} & 0 \\
0 & I_{p}
\end{array}\right], \bar{A}_{i d}=\left[\begin{array}{cc}
A_{i d} & 0 \\
0 & 0
\end{array}\right], \bar{x}=\left[\begin{array}{c}
x^{T} \\
f_{s}^{T}
\end{array}\right], \\
\bar{B}_{i}=\left[\begin{array}{c}
B_{i} \\
0
\end{array}\right], \bar{B}_{i d}=\left[\begin{array}{c}
B_{i d} \\
0
\end{array}\right], \bar{C}_{i}=\left[\begin{array}{c}
C \\
I_{P}
\end{array}\right], f_{s}^{T}(t)=\left[\begin{array}{c}
f_{s 1} \\
f_{s 2} \\
\vdots \\
f_{s i}
\end{array}\right] . \\
R_{i}: \text { If } \xi_{1}(t) \text { is } M_{1 i} \text { and } \xi_{\mathrm{p}}(\mathrm{t}) \text { is } M_{p i} \text {, then } \\
E_{n} \varphi(t)=\sum_{i=1}^{r} q_{i}(\xi(t))\left[\bar{A}_{n i} \varphi(t)+\bar{B}_{i} u(t)\right] \\
\hat{\bar{x}}(t)=\varphi(t)+H y(t)
\end{gathered}
$$

Actually, the states of EMB system are usually unknown, or measurable parts are limited. The estimated values of the states in the T-S fuzzy descriptor nonlinear system are used to realize the fault-tolerant control of sensors. The observer estimating the system states and sensor faults is designed as following equation (4):

where, $\varphi(t)$ is an auxiliary vector of the observer and $\hat{\bar{x}}(t)$ are the state estimations of (1); $H$ is the observer gain to be determined later. Obviously, $\hat{\bar{x}}(t)$ contains the estimation of states and multiple sensor faults.

Define the estimation error as $\bar{e}(t)=\bar{x}(t)-\hat{\bar{x}}(t)$ to obtain the parameters of the state observer. Then, the following equation (5) can be obtained based on equation (2) and equation (4),

$$
E_{n} \dot{\bar{e}}(t)=\sum_{i=1}^{r} q_{i}(\xi(t))\left\{\begin{array}{l}
\bar{A}_{n i} \bar{e}(t)+\left(\bar{A}_{i d}+\Delta \bar{A}_{i d}\right) x(t-d) \\
+\Delta \bar{A}_{i} x(t)+\left(\Delta \bar{B}_{i}+\Delta \bar{B}_{i d}\right) \mu(\tau) \\
+\left[B_{i d}+\Delta B_{i d}(t)\right] u(t-\tau)
\end{array}\right\}
$$

Here, the parameters are calculated as follows:

$$
\bar{A}_{n i}=\left[\begin{array}{cc}
A_{i} & 0 \\
-C & -I
\end{array}\right], \quad E_{n}=\left[\begin{array}{ll}
I & 0 \\
C & I
\end{array}\right], \quad H=\left(\begin{array}{l}
0 \\
I
\end{array}\right) .
$$

\section{Adaptive General Model Control Based on}

$$
\text { SLiding Model CONTRol (SMC-AGMC) }
$$

In building the fault-tolerant control model, the first step is to propose an effective algorithm. In this section, an appropriate sliding surface is selected, then the algorithm of sliding model control and adaptive generic model control are combined together.

For the adaptive fault-tolerant control of complex controlled system, the generic model control (GMC) is an optimization method, it can use nonlinear system model to make the system output track the reference trajectory, the control algorithm is simple, and robust for model uncertainties. The adaptive generic model control is the improvement based on generic model control. It can use Kalman filter to estimate system variable parameters online, and apply it to the classical generic model control to compensate the effect of the time-varying parameters in EMB system.

Transfer the descriptor nonlinear system with multiple inputs and outputs, the equation (1), to the form as follows:

$$
\begin{aligned}
& x(t)=f(x)+g(x) u(t) \\
& y(t)=h x(t)+f_{s}(t)
\end{aligned}
$$

where, $f(x), g(x)$, and $h(x)$ are the nonlinear functions about $x(t)$ in equation (1); $f_{s}(t)$ are the sensor fault vectors; and the relative order of output $y(t)$ is 1 . In classical optimal control, the trajectory of output $y(t)$ is usually compared with some nominal reference trajectory as a system performance index. Having considered the speediness and unbiased property of the system, in order to get the satisfied output response, the reference trajectory of output is defined as follows:

$$
\begin{aligned}
y_{r}(t) & =y^{(n)}(t)+k_{r 0} y^{(n-1)}(t)+\cdots+k_{2} \dot{y}(t) \\
& =K_{1}\left[y_{r}(t)-y_{s p}(t)\right]+K_{2} \int_{0}^{t}\left[y_{r}(t)-y_{s p}(t)\right] d t
\end{aligned}
$$

where, $y_{s p}$ is the setting output. By choosing suitable matrix $K_{1}$ and $K_{2}$, the reference trajectory $y_{r}$ can be adjusted 
automatically. Then, a satisfy system output can be got. By choosing control input $u(t)$, the system output can be made to track the reference trajectory as much as possible. From equation (7),

$$
\begin{aligned}
y^{(n)}(t)= & K_{1}\left[y_{r}(t)-y_{s p}(t)\right]+K_{2} \int_{0}^{t}\left[y_{r}(t)-y_{s p}(t)\right] d t \\
& -k_{r 0} y^{(n-1)}(t)-\cdots-k_{2} \dot{y}(t)
\end{aligned}
$$

Sliding variable structure control is an especial nonlinear control. The best advantage of this sliding structure is its perfect adaptability for disturbances and parameter uncertainties, as well as, the system output can approach to the setting output rapidly when system states enter a sliding control surface. Therefore, the combination of SMC and AGMC can improve the robustness of the nonlinear controlled system. In SMC, $\dot{e}_{y 1}(t)=\dot{y}(t)-\dot{y}_{r}(t)$ is obtained when the model tracking error is set as $e_{y 1}(t)=y(t)-y_{r}(t)$. Then, the error matrix can be defined as $E_{1}=\left[\begin{array}{ll}e_{y 1}(t) & \dot{e}_{y 1}(t)\end{array}\right]^{T}$.

In order to realize the variable structural control target, the controlled system need to have an ideal sliding structure, good dynamic performance, fine robustness. All of those can be achieved by choosing appropriate sliding surface. Then, setting the sliding surface in SMC-AGMC is $s_{1}(t)$,

$$
s_{1}(t)=k_{1} e_{y 1}(t)+k_{2} \int_{0}^{t} e_{y 1}(\tau) d \tau
$$

According to SMC method, the sliding control law is set as $\dot{s}_{1}(t)=-\rho \operatorname{sgn}\left(s_{1}(t)\right)$. From equation (9), it can get as:

$$
\dot{s}_{1}(t)=k_{1} \dot{e}_{y 1}(t)+k_{2} e_{y 1}(t)
$$

To get the corresponding sliding control law, transfer the equation (10) as follows:

$$
\begin{aligned}
\dot{s}_{1}(t) & =k_{1} \dot{e}_{y 1}(t)+k_{2}\left[y(t)-y_{r}(t)\right] \\
& =k_{1} \dot{e}_{y 1}(t)-k_{2} y_{r}(t)+k_{2} C_{i}\left(f(x)+g(x) u_{1}(t)\right) \\
& =-\rho \operatorname{sgn}\left(s_{1}(t)\right)
\end{aligned}
$$

Finally, the control law in general SMC-AGMC is obtained as:

$$
u_{1}(t)=\frac{k_{2} y_{r}(t)-k_{1} \dot{e}_{y 1}(t)-\rho \operatorname{sgn}\left(s_{1}(t)\right)-k_{2} C_{i} f(x)}{k_{2} C_{i} g(x)}
$$

The stability analysis in general SMC-AGMC method is proofed as follows:

From equation (11) and Lyapunov function $V=\frac{1}{2} s^{2}$, it can get as:

$$
\begin{aligned}
\dot{V} & =s_{1}(t) \dot{s}_{1}(t) \\
& =s_{1}(t)\left[-\rho \operatorname{sgn}\left(s_{1}(t)\right)\right] \\
& =-\rho\left|s_{1}(t)\right| \\
& \leq 0
\end{aligned}
$$

Similarly, the system output $y(t)$ in general SMC-AGMC method is become invalid when any of sensors is detected has a fault. Then, replace the output $y(t)$ in the SMC-AGMC calculation by the soft measurement output $\hat{y}(t)$ which is obtained from state observer to realize the SMC-AGMC calculation method. Set the reference model output as $y_{c k}$, then, the model tracking error is set as $e_{y 2}(t)=\hat{y}(t)-y_{r}(t)$. That is $\dot{e}_{y_{2}}(t)=\dot{\hat{y}}(t)-\dot{y}_{r}(t)$. Set the error matrix as $E_{2}=\left[e_{y 2}(t) \dot{e}_{y 2}(t)\right]^{T}$, then, the sliding surface in the state estimation and output prediction based on SMC-AGMC method can be designed as $s_{2}(t)=k_{1} e_{y 2}(t)+k_{2} \int_{0}^{t} e_{y 2}(\tau) d \tau$. Therefore, the corresponding sliding control law is designed as:

$$
u_{2}=\frac{k_{2} y_{r}(t)-k_{1} \dot{e}_{y 2}(t)-\rho \operatorname{sgn}\left(s_{2}(t)\right)-k_{2} C_{i} f(x)}{k_{2} C_{i} g(x)}
$$

In the case of detects fault, the proof of the stability by the sliding model control method is same as equation (13).

\section{The Control Law ReCOnfiguration LogiC}

The fault-tolerant system based on unknown faults must has a fault detection and isolation algorithm, which can judge whether each sensor in EMB system has fault or not according to the estimated values obtained from the designed observer. Fig. 1 shows a control law reconfiguration when sensor fault occurs. First, estimate the sensor fault vectors, $f_{s 1}, f_{s 2}$, and $f_{s 3}$, by state observer. Then, use the estimation values of $f_{s 1}, f_{s 2}$, and $f_{s 3}$ to construct the fault detection and isolation algorithm. Finally, the control law reconfiguration logic is constructed as shown in Fig. 1. As a result, the corresponding fault-tolerant logic will be gotten when the sensor fault is detected. In Fig. $1, \varepsilon_{1}, \varepsilon_{1}^{\prime}, \varepsilon_{2}, \varepsilon_{2}^{\prime}$, and $\varepsilon_{3}^{\prime}$ are threshold values defined before.

If the condition satisfies $\left|\hat{f}_{s 1}\right|>\varepsilon_{1}$, it will be considered that the sensor 1 has a fault. Then, the control law will be switched to the state estimation and output prediction of SMC-AGMC until $\left|\hat{f}_{s 1}\right|<\varepsilon_{1}^{\prime}$. If $\left|\hat{f}_{s 1}\right|<\varepsilon_{1}$, the sensor 1 is considered has no fault, then according $\left|\hat{f}_{s 2}\right|$ to adjust the sensor 2 has fault or not and so on. Where, $\varepsilon_{1}, \varepsilon_{1}^{\prime}, \varepsilon_{2}, \varepsilon_{2}^{\prime}$, $\cdots, \varepsilon_{i}$, and $\varepsilon_{i}^{\prime}$ are set thresholds. According to the proposed reconfiguration control law, the multiple sensors could be judged whether it has faults or not. The faulty sensor will be isolated after the sensor be detected has fault. Then, the adaptive fault-tolerant control law will be switch to the state estimation and output prediction of SMC-AGMC automatically.

\section{Fault-Tolerant Control Architecture Model}

Currently, the researches about the adaptive fault-tolerant control for complex controlled system are more focus on algorithms, there are few researches for build the fault-tolerant control architecture model, especially lack of the variable structure and variable parameter fault-tolerant 
control architecture model. In this section, apply the state observer to the designed sliding adaptive generic model, and build the decision model with dynamic fault reconfiguration, then achieve the goal of the adaptive robust fault-tolerant control for EMB system.

The Kalman filter in traditional adaptive generic model can't satisfy the robust demand against to the model error. Since, the control effect is not enough too. In order to adapt the multiple sensor states, like normal, gradual fault, abrupt fault, and others, the adaptive fault-tolerant control architecture model is proposed as shown in Fig. 2.

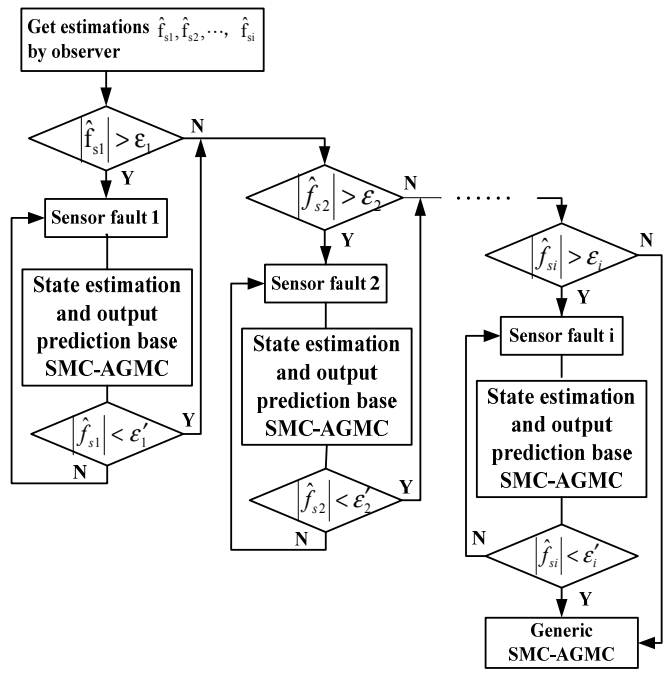

Fig. 1. The control law reconfiguration when any sensor fault occurs.

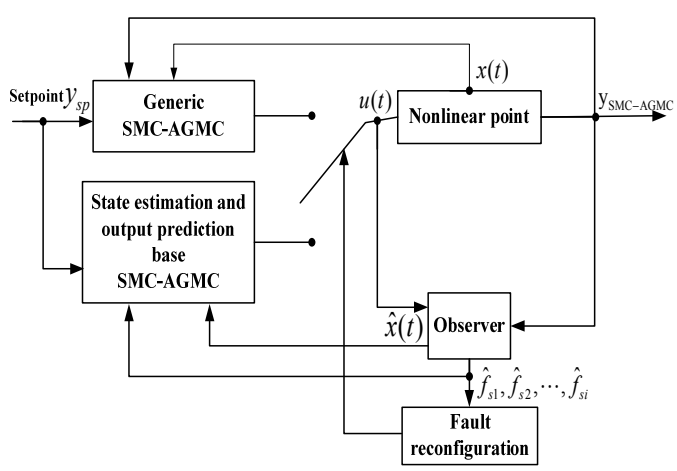

Fig. 2. Fault-tolerant control architecture model.

The main idea of the fault-tolerant control system is shown in Fig. 2. The operating rule of this model is as follows. First, the state observer (4) is used to estimate system states and sensor faults online to get the estimation values $\hat{x}(t)$ and $\hat{f}_{s 1}$, $\hat{f}_{s 2}, \cdots, \hat{f}_{s i}$. The detection and isolation algorithm will be build on the basis of these sensor fault estimation values. Then, the calculating method will be decided according to the control law reconfiguration shown in Fig. 1 as well as the sensor fault. For example, the generic SMC-AGMC algorithm method, output prediction of SMC-AGMC method, or the cutover process. Select the suitable system input, till get the satisfied system output.

\section{Simulation Results}

In order to test the effectiveness of the proposed fault-tolerant control strategy in this paper, numerical
MATALB simulations are conducted to prove the effectiveness of the proposed method. Assuming there are three sensors in the nonlinear system, then the parameters of the nonlinear system (1) with time-delays and parameter uncertainties are taken as follows:

$$
\begin{aligned}
& E=\left[\begin{array}{lll}
1 & 0 & 0 \\
0 & 1 & 0 \\
0 & 0 & 1
\end{array}\right], A_{1}=\left[\begin{array}{ccc}
0 & -1 & 0 \\
1 & -2 & 0 \\
0 & 0 & -1
\end{array}\right], B_{1}=B_{2}=\left[\begin{array}{c}
-1 \\
1 \\
-1
\end{array}\right], \\
& A_{2}=\left[\begin{array}{ccc}
0 & -2 & 0 \\
1 & -3 & 0 \\
0 & 0 & -1
\end{array}\right], B_{11}=B_{21}=\left[\begin{array}{l}
0 \\
1 \\
0
\end{array}\right], E_{11}=\left[\begin{array}{c}
0.2 \\
0 \\
0
\end{array}\right], \\
& A_{11}=A_{12}=\left[\begin{array}{ccc}
0.4 & 0 & 0 \\
0 & 0.1 & -0.1 \\
0 & 0 & 0.5
\end{array}\right], C=\left[\begin{array}{ccc}
-1 & 0 & 0 \\
0 & -1 & 0 \\
0 & 0 & 1
\end{array}\right], \\
& E_{31}=\left[\begin{array}{c}
0.1 \\
0 \\
0
\end{array}\right]^{T}, q_{1}\left(x_{1}(t)\right)=1-\frac{1}{1+e^{-2 x_{1}}}, d=1, \tau=1, \\
& q_{2}\left(x_{1}(t)\right)=\frac{1}{1+e^{-2 x_{1}}}, E_{21}=0.1, E_{41}=0.2 .
\end{aligned}
$$

where, the initial setting of the system is $x_{0}=[1 ; 0 ; 1]$, the setting output is $y_{s p}=0.5$, as well as the parameters in SMC-AGMC control method are $\varepsilon_{1}=0.2, \varepsilon_{1}^{\prime}=3.75$, $\varepsilon_{2}=0.15, \varepsilon_{2}^{\prime}=1.8, \varepsilon_{3}=0.2, \varepsilon_{3}^{\prime}=0.8$, and $k_{1}=2$, $k_{2}=0.8$.

Fig. 3 shows the system states $x_{1}, x_{2}, x_{3}$, and the estimations obtained from the state observer. In Fig.3, the system states are estimated respectively, and the trajectory of the state estimations match with the states of systems in $6 \mathrm{~s}$, this illustrate that the designed observer has fast adjust time and can satisfy the goal to make the closed-loop system to achieve robust stability.

Fig. 4 shows the simulation result of the system sensor fault $f_{s}(t)$ and the estimated sensor fault $\hat{f}_{s}(t)$ by observer (4). In order to test the generic and effectiveness of the designed observer, the abrupt sensor faults and gradual sensor faults are considered into the MATLAB simulation experiments respectively. $f_{s 1}$ and $f_{s 2}$ represent the abrupt sensor faults, and $f_{s 3}$ represents the gradual sensor faults. As shown in Fig. 4, on the both case of abrupt sensor faults and gradual sensor faults, the proposed state observer of T-S fuzzy model shows an excellent estimating ability.

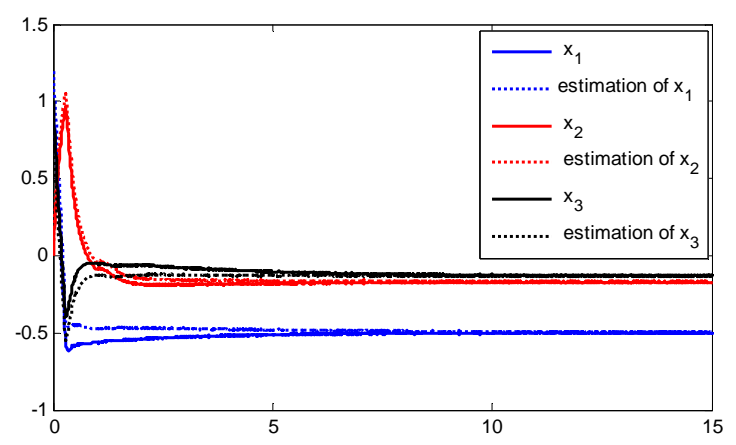

Fig. 3. Model states and estimations.

Fig. 5 shows the system output y-SMCAGMC of the 
adaptive fault-tolerant decision model containing sliding model control, the reference model output $y_{c k}$, and the setting output $y_{s p}$. The output of system and the reference trajectory match well. Finally, it will be consistent with specified output. That is, the EMB system can get satisfy output when sensor faults occur. Meanwhile, there is an integral term in the adaptive generic model control algorithm based on sliding model control, which can offset the influence of the system errors to some extent, so as to achieve the purpose of improving the robustness of the EMB system.

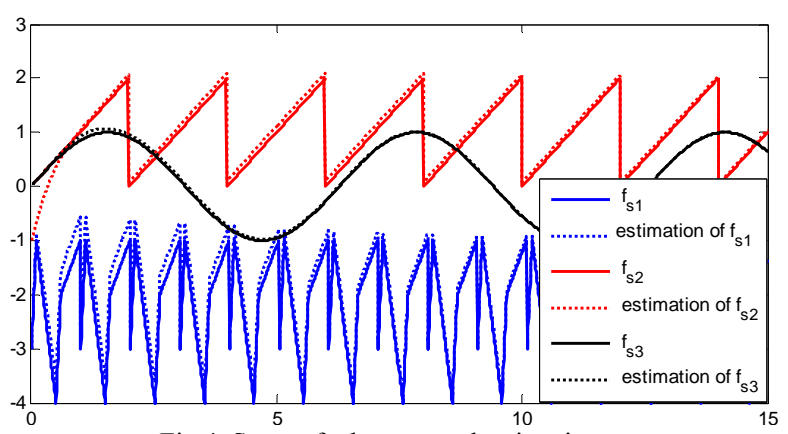

Fig.4. Sensor fault $f_{s}(t)$ and estimations.

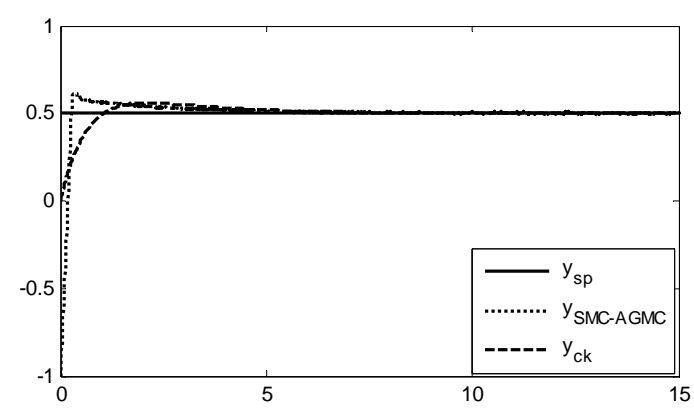

Fig. 5. Model output $\mathrm{y}_{\mathrm{SMC}-\mathrm{AGMC}}$, reference output $\mathrm{y}_{\mathrm{ck}}$ and setting output $\mathrm{y}_{\mathrm{sp}}$.

All the simulation results show that the designed adaptive robust fault-tolerant control dynamic decision model based on T-S fuzzy model which has good robustness for system parameter uncertainties and external interference, it also can estimate the system state and faults in real-time, shorten the time of fault detection. Also, the fact that the system output is consistent with the specified output will maintain a normal work of the EMB system. It demonstrates that adaptive fault-tolerant control based on T-S fuzzy model is effective for EMB system.

\section{CONCLUSION}

In this paper, the T-S fuzzy model is used to describe the nonlinear descriptor system which contains double time-delays, parameter uncertainties, and multiple sensor faults. Next, the state observer is designed for the purpose of estimating system states and sensor faults, and the corresponding fault detection system is constructed. Then, the adaptive generic model control (AGMC) structure is proposed based on sliding model control(SMC). Finally, the whole dynamic decision model of SMC-AGMC with state observer is constructed. The effectiveness and the feasibility of the proposed adaptive fault-tolerant control method were demonstrated through simulations. The dynamic decision model shows a good real-time and the robustness for parameter uncertainties. This nonlinear fault-tolerant control strategy is suitable for systems with multi-sensors where functions of the electronic control systems were realized by these sensors, like the EMB system.

\section{REFERENCES}

[1] R. He, J. Li, Y. D. Li, C. C. Huang, and Q. Wei, " Fault detection approach to EMB sensors based on dediceted observers," in Proc. 2011 International Conference on Electric Information and Control Engineering, 2011, pp. 3266-3269.

[2] W. Hwang, I. Yang, and K. Han, "Model-based sensor fault detection algorithm for EMB system," Transactions of KSAE, vol. 20, pp. 1-7, July 2011.

[3] Y. Q. Wang, D. H. Zhou, and L. H. Liu, "Robust and active fault-tolerant control for a class of nonlinear uncertain systems," International Journal of Automation and Computing, vol. 3, pp. 309 313, February 2006.

[4] B. Jang, Z. F. Gao, P. Shi, and Y. F. Xu, "Adaptive fault-tolerant tracking control of near-space vehicle using Takagi-Sugeno fuzzy models," IEEE Transactions on Fuzzy Systems, vol. 18, pp. 1000-1007, October 2010

[5] Z. W. Gao, T. F. Breikin, and H. Wang, "Reliable observer-based control against sensor failures for systems with time delays in both state and input," IEEE Trans. Systems Man Cybernetics, vol. 38, pp. 1018-1029, August 2008.

[6] D. Wang, D. H. Zhou, Y. H. Jin, and S. J. Qin, "Adaptive generic model control for a class of nonlinear time-varying processes with input time delay," Journal of Process Control, vol. 14, pp. 517-531, August 2004.

[7] M. C. Pai, "Observer-based adaptive sliding mode control for nonlinear uncertain state-delayed systems, International Journal of Control, Automation, and Systems, vol. 7, pp. 536-544, August 2009.

[8] H. L. Xing, C. C. Gao, D. H. Li, "Sliding model variable structure control for parameters uncertain stochastic systems with time-varying," Mathematical Problems in Engineering, p. 8, 2008.

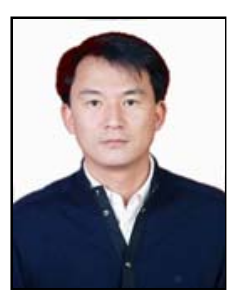

Y. N. Xu is an associate professor of the division of electronic and communication engineering of Yanbian University, Yanji, China. He received the $\mathrm{Ph} . \mathrm{D}$. degree in electrical engineering from the Chonbuk National University, Korea, in 2009. His research interests include the In-vehicle networks and automobile electronic control.

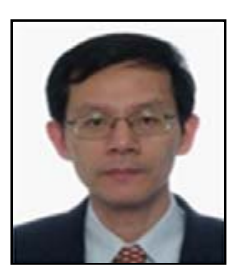

W. W. Deng is a professor of the State Key Laboratory of Automotive Simulation and Control, Jilin University, Changchun, China. He has been working for General Motors R\&D Center since 1996. He holds BSc and MSc degrees in Mechanical Engineering from China, and and $\mathrm{Ph}$.D. in electrical engineering from USA. He is a staff researcher at GM and the twice recipients of the 'Boss' Kettering Award, the most prestigious award in GM for technical invention and innovation. He holds 17 US patents with another 16 pending, and is the author of over 30 journal and conference publications. Currently, he also serves as Editor or Associate Editor for several international journals. 